Radiologe 2020 60:934-942

https://doi.org/10.1007/s00117-020-00743-w

Online publiziert: 28 . August 2020

(c) Springer Medizin Verlag $\mathrm{GmbH}$, ein Teil von Springer Nature 2020
Bereits im Mai 2020 diskutierten Gattinoni et al., dass einige Patienten mit COVID-19 und akutem hypoxämischem Atemversagen eine relativ stabile Lungencompliance beibehalten [1]. Diese Ergebnisse deuten darauf hin, dass neben der mit einem akuten Alveolarschaden in Zusammenhang stehenden Diffusionsstörung weitere Prozesse an der bei Patienten mit schwerer COVID-19 beobachteten Hypoxämie beteiligt sein könnten. Darüber hinaus wurden zwei theoretische Phänotypen abgegrenzt: der L-Typ, der von einer niedrigen Elastance und einem niedrigen Ventilation-zuPerfusions-Verhältnis geprägt ist, und der H-Typ, der eine hohe Elastance und einen hohen Rechts-Links-Shunt aufweist, woraus unterschiedliche Behandlungsstrategien abgeleitet wurden [2]. Zeitgleich häuften sich Berichte zu ,außergewöhnlich häufigen “ thromboembolischen Ereignissen bei hospitalisierten COVID-19-Patienten, woraus trotz zunächst fehlender randomisierter Evidenz eine frühzeitige, intensivierte Thromboseprophylaxe für Intensivpatienten mit

W. L. Wagner, ${ }^{1,2} \cdot$ K. Hellbach ${ }^{1,2} \cdot$ M. O. Fiedler ${ }^{2,3} \cdot$ G. A. Salg ${ }^{4} \cdot$ E. Wehrse ${ }^{5,6} \cdot$

C. H. Ziener ${ }^{2,5} \cdot$ U. Merle ${ }^{7}$. C. Eckert ${ }^{8} \cdot$ T. F. Weber ${ }^{1} \cdot$ W. Stiller ${ }^{1,2} \cdot$ M. O. Wielpütz ${ }^{1,2}$.

C. Dullin ${ }^{9} \cdot$ H. G. Kenngott ${ }^{4}$ - H.-P. Schlemmer ${ }^{2,5} \cdot$ M. A. Weigand ${ }^{2,3}$.

P. Schirmacher ${ }^{8,10} \cdot$ T. Longerich ${ }^{8} \cdot$ H.-U. Kauczor ${ }^{1,2} \cdot$ F. K.-F. Kommoss ${ }^{8} \cdot$ C. Schwab ${ }^{8}$

' Klinik für Diagnostische und Interventionelle Radiologie, Universitätsklinikum Heidelberg, Heidelberg, Deutschland

${ }^{2}$ Zentrum für Translationale Lungenforschung (TLRC), Deutsches Zentrum für Lungenforschung (DZL), Universitätsklinikum Heidelberg, Heidelberg, Deutschland

${ }^{3}$ Klinik für Anästhesiologie, Universitätsklinikum Heidelberg, Heidelberg, Deutschland

${ }^{4}$ Neue Technologien und Datenwissenschaften/3D-Biodruck Einheit, Klinik für Allgemein-, Viszeral-, und Transplantationschirurgie, Universitätsklinikum Heidelberg, Heidelberg, Deutschland

${ }^{5}$ Abteilung Radiologie, Deutsches Krebsforschungszentrum (DKFZ), Heidelberg, Deutschland

${ }^{6}$ Medizinische Fakultät, Ruprecht-Karls-Universität Heidelberg, Heidelberg, Deutschland

${ }^{7}$ Klinik für Gastroenterologie, Infektionen, Vergiftungen, Universitätsklinikum Heidelberg, Heidelberg, Deutschland

${ }^{8}$ Pathologisches Institut, Universitätsklinikum Heidelberg, Heidelberg, Deutschland

${ }^{9}$ Institut für Diagnostische und Interventionelle Radiologie, Universitätsmedizin Göttingen, Göttingen, Deutschland

${ }^{10} \mathrm{TI}$ Biobank, Deutsches Zentrum für Infektionsforschung (DZIF), Universitätsklinikum Heidelberg Heidelberg, Deutschland

\title{
Mikrovaskuläre Veränderungen bei COVID-19
}

COVID-19 abgeleitet wurde [3]. Mit der Veröffentlichung erster Obduktionsreihen an COVID-19 verstorbenen Patienten konnten erste pathophysiologische Erklärungsansätze für diese Beobachtungen aufgezeigt werden: Ackermann et al. konnten eine Endothelialitis kleinster pulmonaler Blutgefäße, eine im Vergleich zur Influenzapneumonie und einer nichtinfizierten Kontrollgruppe erhöhte Mikrogefäßthrombosierung und konsekutiv eine gesteigerte Angiogenese nachweisen [4]. Eine räumlichzeitliche Entwicklung der pulmonalen und mikrovaskulären Schädigungsmuster wurde anhand von topographischer Läsionsverteilung, Erkrankungsdauer und Dauer der invasiven Beatmung durch Kommoss et al. abgeleitet [5]; aus letzterer Obduktionskohorte entstammen auch die zwei hier im Detail präsentierten Fälle.

Der radiologischen Bildgebung vor allem in Form der Computertomographie (CT) des Thorax - kommt in der Diagnostik bei Patienten mit V.a. COVID-19-Pneumonie und in der Ver- laufsbeurteilung bei Verschlechterung der respiratorischen Symptomatik bestätigter COVID-19-Patienten eine entscheidende Rolle zu [6]. Hierbei lassen sich typische durch die SARS-CoV-2Infektion hervorgerufene radiologische Muster abgrenzen, die den Verdacht auf eine COVID-19-Lungenerkrankung erhärten oder entkräften können. Zudem kann die Bildgebung in der Differenzialdiagnostik wegweisend sein. Wenn differenzialdiagnostisch z.B. der Verdacht auf eine Lungenarterienembolie besteht, sollte die CT-Untersuchung kontrastmittelgestützt erfolgen. Die DualEnergy- bzw. Spektral-CT-Bildgebung kann dann zur Charakterisierung der Lungenperfusion verwendet werden [7]. In diesem Kontext berichteten Lang et al. bereits von auffälligen Perfusionsanomalien: Zusätzlich zu den typischen CTMerkmalen einer COVID-19-Pneumonie wurden „deutliche Dilatationen und Tortuositäten“ der Lungengefäße innerhalb oder in der Umgebung von COVID19-typischen Läsionen beschrieben. Zudem wurde von deutlich reduzierter 


\begin{tabular}{|c|c|c|c|c|c|c|c|c|}
\hline Patient & Alter & Geschlecht & BMI & $\begin{array}{l}\text { Nikotin- } \\
\text { abusus }\end{array}$ & Komorbidität & Symptome & $\begin{array}{l}\text { Erkrankungs- } \\
\text { dauer (Tage) }\end{array}$ & $\begin{array}{l}\text { Krankenhaus- } \\
\text { aufenthalt (Tage) }\end{array}$ \\
\hline 1 & 71 & $\mathrm{~m}$ & 24,3 & $\varnothing$ & KHK & Fieber $40^{\circ} \mathrm{C}$ & 34 & 30 \\
\hline 2 & 76 & $\mathrm{~m}$ & 27,1 & n. b. & $\begin{array}{l}\text { NSTEMI, AH, } \\
\text { DM, KHK }\end{array}$ & $\begin{array}{l}\text { Fieber } 39^{\circ} \mathrm{C} \text {, Dyspnoe, Abge- } \\
\text { schlagenheit }\end{array}$ & 40 & 33 \\
\hline
\end{tabular}

\begin{tabular}{|c|c|c|c|c|}
\hline Patient & $\begin{array}{l}\text { Medikamentöse } \\
\text { Therapie }\end{array}$ & $\begin{array}{l}\text { Invasive Beatmung } \\
\text { (h) }\end{array}$ & $\begin{array}{l}\text { Invasive Beatmung MW PEEP } \\
(\mathrm{mm} \mathrm{Hg})\end{array}$ & $\begin{array}{l}\text { Invasive Beatmung MW Compliance } \\
\text { (ml/mbar) }\end{array}$ \\
\hline 1 & $A B, A V, I M T, V H$ & 486 & 10,102 & 46,247 \\
\hline 2 & $A B, A V, I M T, V H$ & 643 & 8,263 & 91,097 \\
\hline
\end{tabular}

\begin{tabular}{|l|l|l|l|l|l|l|}
\hline $\begin{array}{l}\text { Tab. 3 } \\
\text { Patient }\end{array}$ & $\begin{array}{l}\text { Klinische CT-Bildgebung } \\
\text { Colta zum } \\
\text { Todeszeitpunkt } \\
\text { (Tage) }\end{array}$ & $\begin{array}{l}\text { Untersuchungs- } \\
\text { protokoll }\end{array}$ & $\begin{array}{l}\text { COVID-19-typische } \\
\text { Lungenveränderun- } \\
\text { gen }\end{array}$ & $\begin{array}{l}\text { Dominante Muster der } \\
\text { COVID-19-typischen Verän- } \\
\text { derungen }\end{array}$ & $\begin{array}{l}\text { Pulmonale } \\
\text { Perfusions- } \\
\text { anomalien }\end{array}$ & $\begin{array}{l}\text { Führender Ab- } \\
\text { domenbefund }\end{array}$ \\
\hline 1 & 5 & $\begin{array}{l}\text { KM CT Th/Abd multi- } \\
\text { phasisch Multi-Energy }\end{array}$ & Ja & Milchglas $>$ Konsolidierungen & Ja & $\begin{array}{l}\text { Intestinale } \\
\text { Passagestörung }\end{array}$ \\
\hline 2 & 0 & $\begin{array}{l}\text { KM CT Th/Abd multi- } \\
\text { phasisch Multi-Energy }\end{array}$ & Ja & Milchglas $>$ Retikulationen & Nein & $\begin{array}{l}\text { Mesenteriale } \\
\text { Ischämie }\end{array}$ \\
\hline KMCT Th/Abd kontrastmittelgestützte Computertomographie Thorax/Abdomen & & \\
\hline
\end{tabular}

Tab. 4 Zellpopulationsspezifische ACE2-Expression mit funktionellen Implikationen

\begin{tabular}{lll}
\hline Zellpopulation & ACE2-Expression & Funktionelle Implikation \\
\hline Pneumozyten Typ I & Hoch $[10,11]$ & Blut-Luft-Schranke \\
\hline Pneumozyten Typ II & Hoch $[10,11]$ & Alveoläre Mikromechanik und Regeneration \\
\hline Pulmonale Endothelzellen & Hoch $[10,11]$ & Blut-Luft-Schranke, Vasoregulation \\
\hline Perizyten & Hoch $[12-14]$ & Vasoregulation \\
\hline ACE2 Angiotensin-Converting Enzyme 2 & \\
\hline
\end{tabular}

Perfusion innerhalb der Läsionen und gesteigerter Perfusion proximal und um die Läsionen berichtet [7].

In diesem Artikel werden anhand von zwei exemplarischen Fällen Ausprägungsformen der Mikrovaskulopathie im Zusammenhang mit COVID19 präsentiert. Beide Patienten zeigten Perfusionsauffälligkeiten in der kontrastmittelgestützten Spektral-CT von Thorax und Abdomen, zu denen eine gezielte mikroradiologische und pathologische Korrelation anhand der Obduktionspräparate erfolgte.

\section{Material und Methoden}

Alle organisatorischen und räumlichen Vorsichtsmaßnahmen, die zum
Personal- und Infektionsschutz notwendig sind, wurden berücksichtigt. Klinische CT-Untersuchungen erfolgten an einem Dual-layer-CT-System mit 64 Detektorzeilen (iQon Spectral CT, Philips Healthcare, Best, Niederlande). Zur Kontrastverstärkung wurde ein Hochdruckinjektor verwendet, um ein Kontrastmittelvolumen von $95 \mathrm{ml}$ (Accupaque $350 \mathrm{mgI} / \mathrm{ml}$; GE Healthcare, Braunschweig, Deutschland) mit einer Flussrate $\geq 3,0 \mathrm{ml} / \mathrm{s} \mathrm{zu}$ injizieren. Die Durchführung der Obduktionen erfolgte nach einem standardisierten Verfahren und wurde detailliert bereits an anderer Stelle beschrieben [5].Zusammenfassend wurden die Lungen nach trachealer Instillation und Spülung der großen Pulmonalgefäße mit $4 \%$ Forma- lin für $>72 \mathrm{~h}$ in $4 \%$ Formalin nachfixiert. Die fotodokumentierte Präparation der Lungen erfolgte in axialer Schichtung zu ca. $1 \mathrm{~cm}$ starken Lamellen. Jeweils direkt angrenzende Lamellen wurden hier zur histopathologischen und mikroradiologischen Untersuchung verwendet. Nach Paraffineinbettung wurden histopathologische Schnittpräparate von Gewebeproben angefertigt und nach Standardprotokollen für Hämatoxylin-EosinFärbung (HE) und saurem FuchsinOrange G (AFOG) gefärbt. Die Trocknung fixierter Lungenlamellen erfolgte durch eine aufsteigende Ethanolreihe, Behandlung mit Hexamethyldisilazan und anschließender Lufttrocknung wie zuvor beschrieben [8]. Die mikroradiologische Bildgebung der getrockneten Lamellen erfolgte mittels eines experimentellen photonenzählenden Ganzkörper-Computertomographen (Somatom CounT, Siemens Healthineers, Forchheim, Germany) im ultrahochauflösenden (UHR) Modus mit einer Pixelgröße von $270 \times 270 \mu \mathrm{m}$ und einer Schichtdicke von $500 \mu \mathrm{m}$ sowie mittels eines Bruker SkyScan 1176 Mikro-Computertomo- 
Radiologe 2020 · 60:934-942 https://doi.org/10.1007/s00117-020-00743-w

(c) Springer Medizin Verlag GmbH, ein Teil von Springer Nature 2020

W. L. Wagner · K. Hellbach · M. O. Fiedler · G. A. Salg · E. Wehrse · C. H. Ziener · U. Merle · C. Eckert · T. F. Weber · W. Stiller · M. O. Wielpütz · C. Dullin · H. G. Kenngott · H.-P. Schlemmer · M. A. Weigand · P. Schirmacher · T. Longerich · H.-U. Kauczor · F. K.-F. Kommoss · C. Schwab

\section{Mikrovaskuläre Veränderungen bei COVID-19}

\section{Zusammenfassung}

Hintergrund. Klinisch ist COVID-19 (Corona Virus Disease 2019) mit einem breiten Spektrum an Symptomen verbunden, das von milden Beschwerden eines oberen Atemwegsinfekts bis zur lebensbedrohlichen hypoxischen respiratorischen Insuffizienz und Multiorganversagen reichen kann.

Fragestellung. Zu den initial identifizierten pulmonalen Schädigungsmustern, wie dem diffusen Alveolarschaden bei akutem Lungenversagen, gesellen sich neue Erkenntnisse, die ein komplexeres Szenario zeichnen. Hierzu gehören Mikrogefäßbeteiligungen und ein breites Spektrum assoziierter Pathologien multipler Organsysteme. Eine Rückskalierung mikrostruktureller Gefäßveränderungen ist durch gezielte Korrelation pathologischer Obduktionsergebnisse mit radiologischer Bildgebung möglich.
Material und Methoden. Radiologischpathologische Korrelation und mikroradiologische Bildgebung zur Untersuchung der Mikrogefäßbeteiligung bei letaler COVID-19. Ergebnisse. Die Fälle zweier COVID-19Patienten werden präsentiert. Patient 1 zeigte eine relative Minderperfusion in Lungenarealen, die radiologisch keine COVID19-typischen Infiltrate aufwiesen; die gezielte autoptische Korrelation ergab auch in diesen Lungenabschnitten bereits subtile Zeichen eines Mikrogefäßschadens. Patient 2 zeigte radiologisch und pathologisch eine fortgeschrittene COVID-19-typische Lungenstrukturdestruktion; der Fall illustriert die Schädigungsmuster der Blut-LuftSchranke. Die CT-morphologisch perfusionsgestörte Darmwand von Patient 2 konnte letztendlich mikroskopisch nicht eindeutig auf einen intestinalen Mikrogefäßschäden zurückgeführt werden.

Schlussfolgerung. Die Ergebnisse weisen neben der Mikrogefäßthrombosierung auf eine funktionelle pulmonale Vasodysregulation im Rahmen der vaskulären Phase von COVID19 hin. Hervorzuheben sind die klinische Relevanz von Obduktionen und eine enge Verzahnung von radiologischen Bildbefunden mit histopathologischen Schädigungsmustern zum besseren Verständnis von COVID-19.

Schlüsselwörter

SARS-CoV-2 - Mikrogefäße · Computertomographie $\cdot$ Perfusion - Pathologische Korrelation

\section{Microvascular changes in COVID-19}

\section{Abstract}

Background. Clinically, coronavirus disease 2019 (COVID-19) is associated with a wide range of symptoms, which can range from mild complaints of an upper respiratory infection to life-threatening hypoxic respiratory insufficiency and multiorgan failure.

Objective. The initially identified pulmonary damage patterns, such as diffuse alveolar damage in acute lung failure, are accompanied by new findings that draw a more complex scenario. These include microvascular involvement and a wide range of associated pathologies of multiple organ systems. A back-scaling of microstructural vascular changes is possible via targeted correlation of pathological autopsy results with radiological imaging.
Material and methods. Radiological and pathological correlation as well as microradiological imaging to investigate microvascular involvement in fatal COVID-19.

Results. The cases of two COVID-19 patients are presented. Patient 1 showed a relative hypoperfusion in lung regions that did not have typical COVID-19 infiltrates; the targeted post-mortem correlation also showed subtle signs of microvascular damage even in these lung sections. Patient 2 showed both radiologically and pathologically advanced typical COVID-19 destruction of lung structures and the case illustrates the damage patterns of the blood-air barrier. The perfusion deficit of the intestinal wall shown in computed tomography of patient 2 could not ultimately clearly be microscopically attributed to intestinal microvascular damage. Conclusion. In addition to microvascular thrombosis, our results indicate a functional pulmonary vasodysregulation as part of the pathophysiology during the vascular phase of COVID-19. The clinical relevance of autopsies and the integration of radiological imaging findings into histopathological injury patterns must be emphasized for a better understanding of COVID-19.

\section{Keywords}

SARS-CoV-2 $\cdot$ Microvessels $\cdot$ Computed tomography · Perfusion - Pathological correlation graphen $(\mu \mathrm{CT})$ mit einer resultierenden Voxelkantenlänge von $9 \mu \mathrm{m}$, wie bereits detailliert an anderer Stelle beschrieben [9]. Die digitale $\mu \mathrm{CT}$-Bilddatenanalyse erfolgte mit den Analyseprogrammen VGSTUDIO Max 3.4.0 (Volume Graphics, Heidelberg, Deutschland) und CTVox 3.1.1 (Bruker Biospin, Rheinstetten, Deutschland).

\section{Ergebnisse}

\section{Patient 1}

Klinische Epikrise. 71-jähriger Mann, der 4 Tage nach Symptombeginn mit hohem Fieber und V.a. COVID-19 stationär aufgenommen wurde. Drei Tage nach Aufnahme wurde mittels eines nasopharyngealen Abstrichs und RTPCR (Reverse-Transkriptase-Polymera-
se-Kettenreaktion) eine Infektion mit SARS-CoV-2 bestätigt. Nach 3-tägiger nichtinvasiver Beatmungstherapie mit nasaler High-flow-Oxygenierungstherapie (HFNO) erfolgte bei respiratorischer Verschlechterung die Intubation und invasive Beatmung für insgesamt $486 \mathrm{~h}$ (- Tab. 1 und 2). Nach einmaliger Extubation erschöpfte sich der Patient respiratorisch bei bestehender Hypoxämie, sodass eine Re-Intubation und 


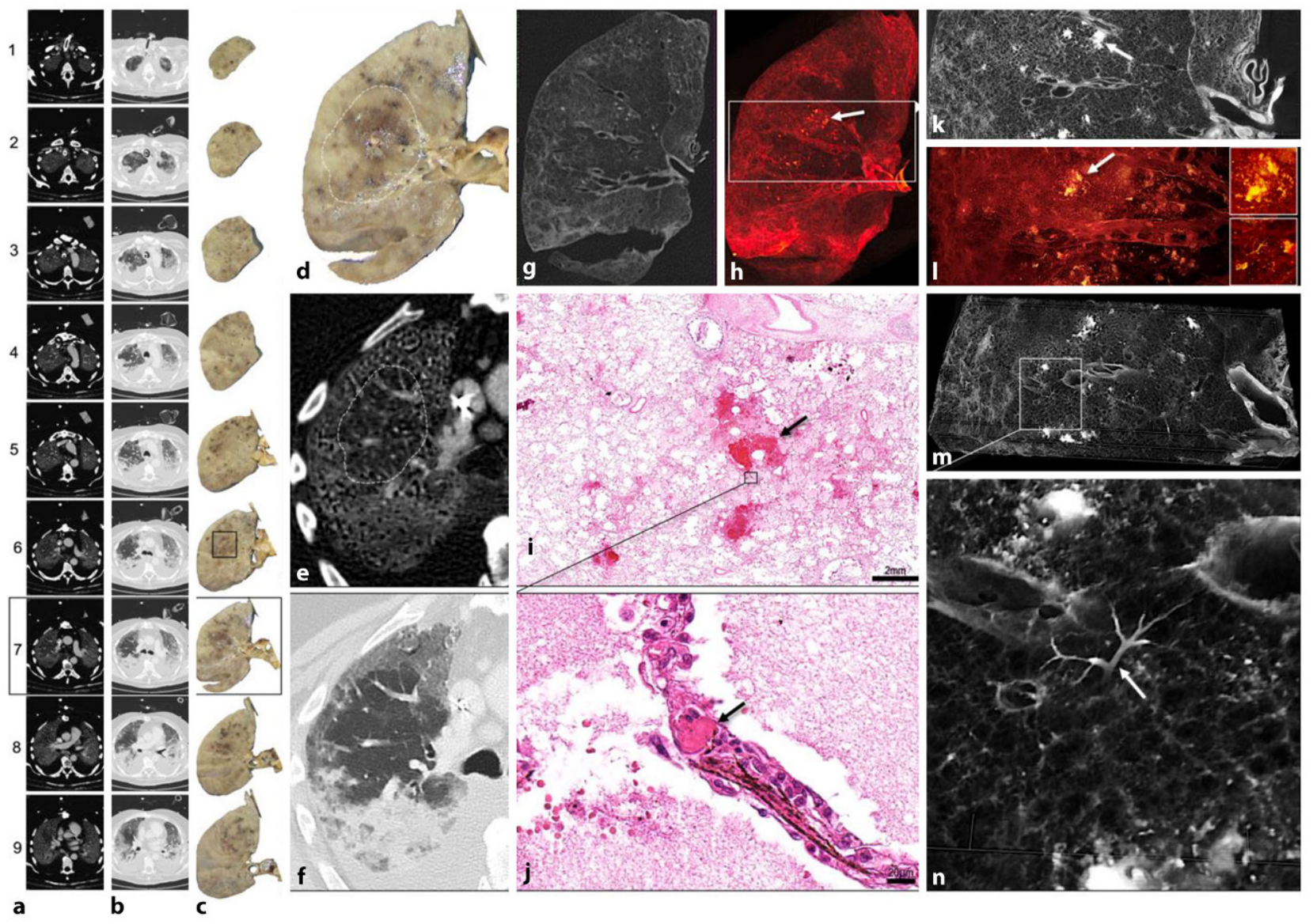

\begin{abstract}
Abb. 1 \& Patient 1. a-c Korrespondierende serielle Schichten der Jodkarte (a), des Lungenfensters (b) sowie die standardisiert präparierten Obduktionslamellen des rechten Oberlappens (c). $\mathbf{d}$-f Lamelle 7 mit korrespondierenden Schichten der CT: ein relativ vermindert kontrastiertes Areal in der Jodkarte (e) korrespondiert mit einem Areal normaler Dichte im Lungenfenster (f) und einem makropathologisch dunkel imponierenden Bereich (d). g, h UHR-Aufnahmen der Lamelle 7 nach chemischer Trocknung im Lungenfenster (g) und in der Volumenrekonstruktion (h) zeigen winzige fokale Verdichtungen im Lungenparenchym, die in Morphologie und Ausdehnung mittels $\mu C T$ exakter zu charakterisieren sind ( $\mathbf{k}, \mathbf{l}$, ergänzendes Video 1). i, j Korrelative Histopathologie aus dem direkt angrenzenden Bereich (6C) zeigt blutgestaute Gefäße und eine fokale alveoläre Hämorrhagie (Pfeil in i) sowie vereinzelte fibrinoide Mikrogefäßthromben. Diese Befunde lassen sich mittels $\mu \mathrm{CT}$ dreidimensional abbilden ( $\mathbf{k}-\mathbf{n}$, ergänzendes Video 1). Skalenintervalle in $(\mathbf{k}-\mathbf{n})=5 \mathrm{~mm}$
\end{abstract}

invasive Beatmung erfolgten; 14 Tage nach Re-Intubation verstarb der Patient.

\section{Radiologische Befunde und histopa- thologische Korrelation. Am 25. Be-} handlungstag, 5 Tage vor dem Versterben des Patienten, erfolgte bei klinischer Verschlechterung mit $V$. a. einen zusätzlichen abdominellen Infektfokus eine kontrastmittelgestützte CT Thorax/Abdomen (• Tab. 3). Es zeigten sich schwere Ausprägungsformen einer COVID-19-Lungenerkrankung mit bilateralen, fleckigen Milchglasinfiltraten, Konsolidierungen, retikulären Zeichnungsvermehrungen und linearen parenchymatösen Verdichtungen ( $\bullet$ Abb. 1b). In der Jodkarte
(• Abb. 1a) zeigte sich zentral im rechten Oberlappen ein relativ minderkontrastiertes Areal ohne Nachweis der o.g. COVID-19-typischen Läsionen im Lungenfenster $(\bullet \mathbf{A b b} .1 \mathrm{1e}, \mathbf{f})$. Zwischen der klinischen CT-Bildgebung und dem Versterben des Patienten lagen $5 \mathrm{Ta}$ ge. Makropathologisch korrelierte das in der CT relativ minderperfundierte Lungenareal mit einem Bezirk weniger konsolidierten Lungenparenchyms von weicherer Konsistenz (• Abb. 1c, d): ein Befund, der auf belüftetes, strukturell weniger geschädigtes Lungengewebe hinweist. Nach Dehydration der Lungenlamelle ließen sich mikroradiologisch ebendort kleinste sphärische Verdich- tungen abgrenzen ( $\bullet$ Abb. 19, h, $\mathbf{k}-\mathbf{n}$ ). Die gezielte histopathologische Korrelation der zentralen Anteile der direkt angrenzenden Lamelle 6 zeigte fokale alveoläre Hämorrhagien und vereinzelte fibrinoide Mikrogefäßthromben in der direkten Umgebung der Einblutungen $(\bullet \mathbf{A b b} . \mathbf{1 i}, \mathbf{j})$. Die alveoläre Parenchymarchitektur war weitgehend erhalten, fortgeschrittene emphysematöse, fibrosierende oder desquamative entzündliche Veränderungen waren nicht nachweisbar. 

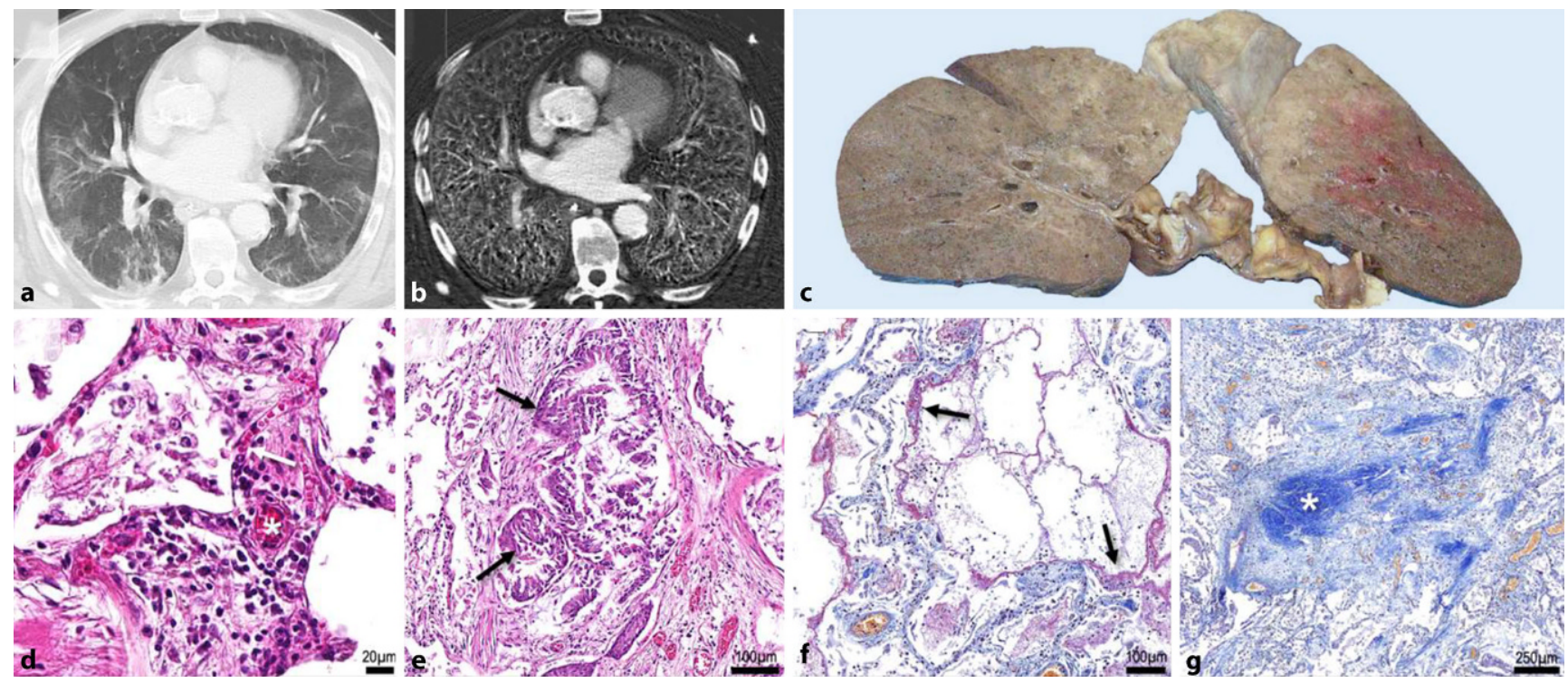

Abb. 2 \ Patient 2 (Lunge). a COVID-19-typische bilaterale, peripher betonte Milchglasverdichtungen, diskrete periphere Retikulierung und minimale Konsolidierung im Lungenfenster der kontrastmittelgestützten Computertomographie (CT) wenige Stunden vor dem Versterben des Patienten. In der Jodkarte sind keine eindeutigen pulmonalen Perfusionsdefekte abgrenzbar (b). Die zentralen rötlichen Areale im linken Unterlappen entsprechen noch unvollständig fixiertem Gewebe (c). Die Lichtmikroskopie zeigt typische Schädigungsmuster der COVID-19-Pneumonie mit fokaler perimikrovaskulärer Lymphozyteninfiltration (d), prominenter Hyperplasie/Metaplasie der Typ-II-Pneumozyten (e), Ausbildung hyaliner Membranen im Alveolarraum (f) und fokaler Fibrosierung (g). Frische Mikrothromben lassen sich in diesem Areal nicht nachweisen
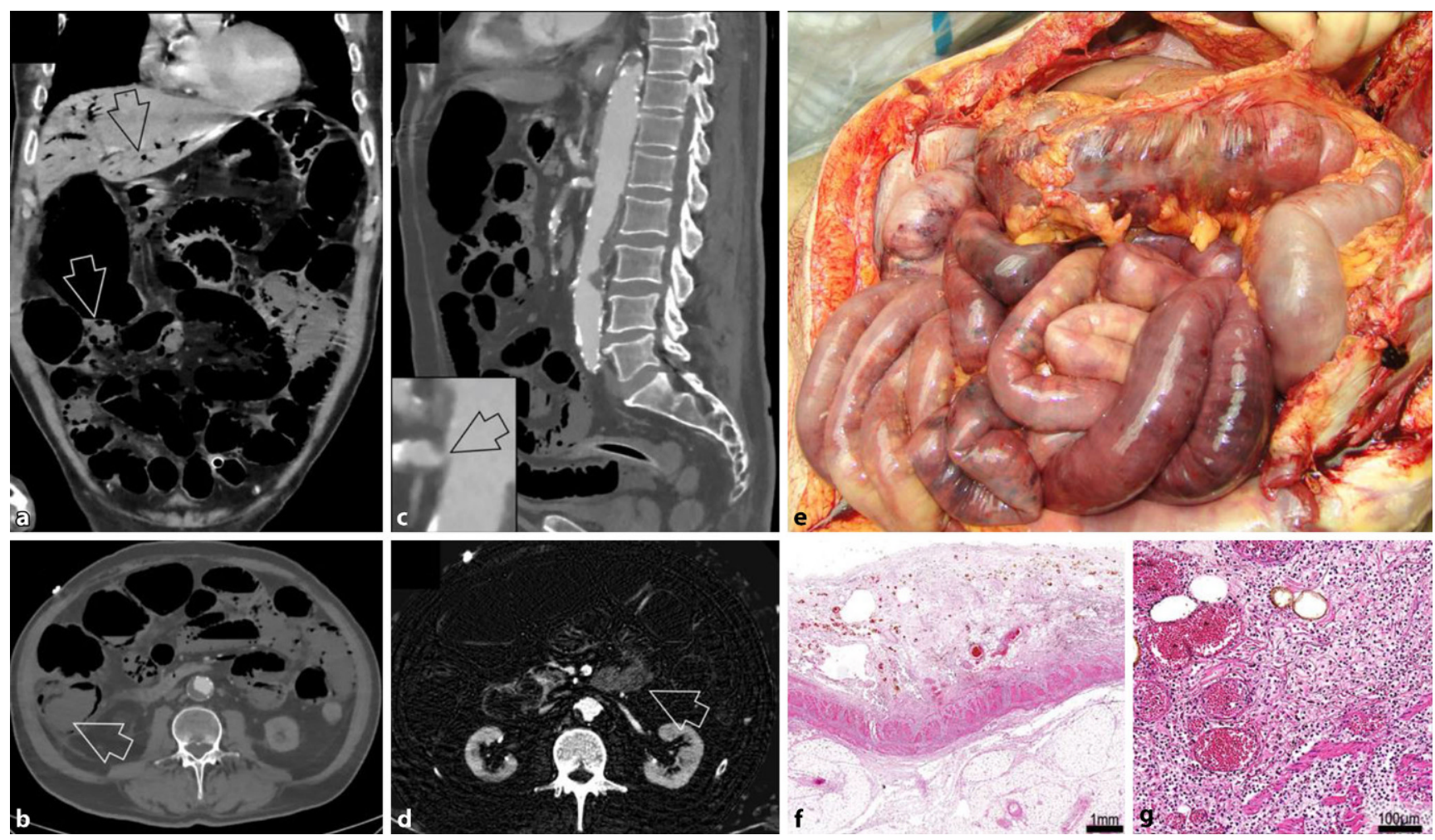

Abb. 3 ॥ Patient 2 (Abdomen). Die multiphasische Computertomographie (CT) des Abdomens zeigt Stigmata einer Mesenterialischämie mit Pneumatosis intramuralis $(\mathbf{a}, \mathbf{b})$ und Pneumatosis portalis (a). Bei subtotaler Abgangsstenose der A. mesenterica superior (c) zeigt sich sonst die arterielle mesenteriale Strombahn erhalten kontrastiert ohne Nachweis thrombembolischer Kontrastmittelaussparungen. In der Jodkarte zeigt sich eine erhaltene Kontrastierung der Duodenalwand bis zum Treitz-Band ohne weitere murale Jodaufnahme des gesamten Dünn- oder Dickdarms (d). Autoptisch bestätigt sich die Ischämie des Dünn- und Dickdarms (e-g) mit Schleimhautnekrose bei Zeichen einer intramuralen Perfusionsstörung 


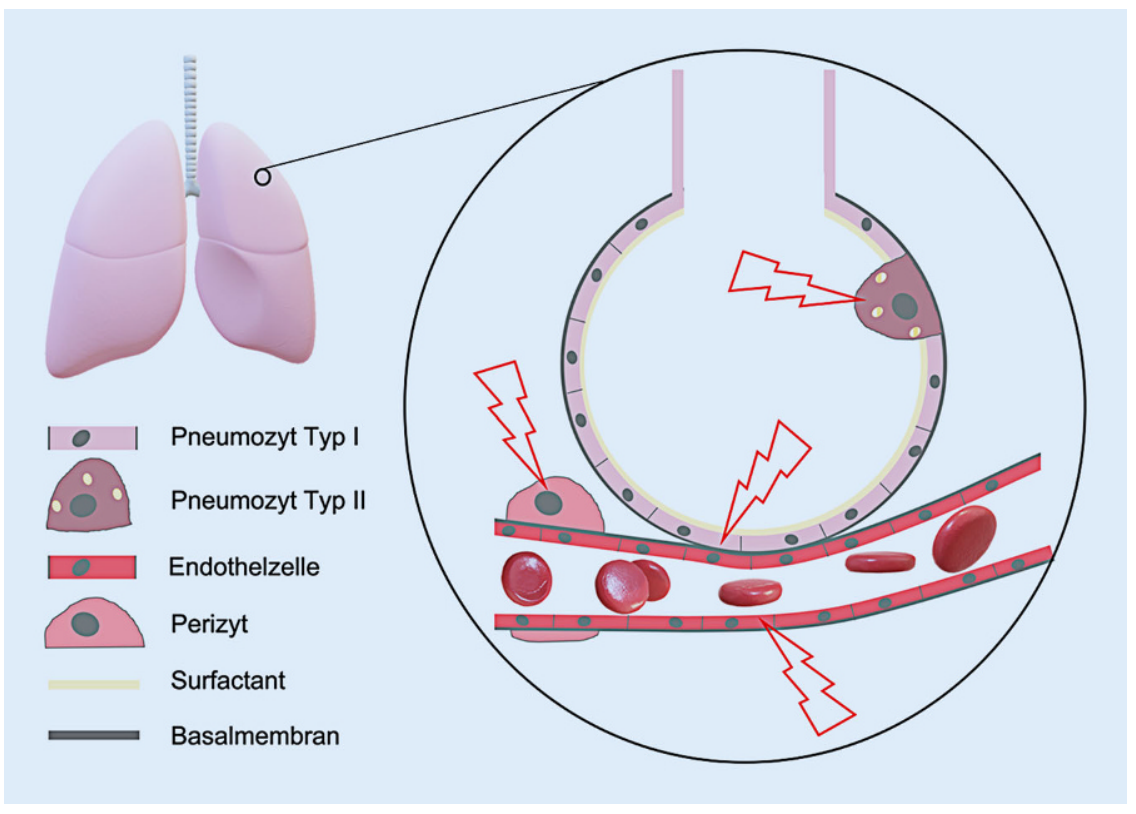

Abb. 4 \ Schematische Darstellung eines pulmonalen Alveolus mit assoziierter Alveolarkapillare. Pneumozyten vom Typ II sind für die alveoläre Surfactantbildung zuständig und somit essenziell für eine physiologische alveoläre Mikromechanik. Darüber hinaus bilden sie den Stammzellpool für die Regeneration der Typ-I-Pneumozyten, die ihrerseits über eine verschmolzene Basalmembran die Blut-Luft-Schranke mit den alveolären Kapillarendothelzellen bilden. Perizyten sind kontraktile gefäßassoziierte Bindegewebszellen, die an der Vasoregulation beteiligt sind. Alle hier aufgeführten Zellpopulationen weisen eine hohe ACE2-Expression auf (funktioneller Rezeptor für Coronaviren; [10-14]) und stellen somit potenzielle Risikostrukturen für eine direkte Schädigung durch das SARS-CoV-2 dar: Eine Annahme, die allerdings weiterer Studien auf ultrastruktureller Ebene bedarf. Ihre Schädigung wird in kausalen Zusammenhang mit den im Artikel beschriebenen strukturellen und funktionellen Schädigungsmustern gebracht

\section{Patient 2}

Klinische Epikrise. 76-jähriger Mann, der eine Woche nach Symptombeginn mit Fieber, Dyspnoe und Abgeschlagenheit stationär aufgenommen wurde. Die Intubation erfolgte am 9. Behandlungstag, der Patient wurde für insgesamt $643 \mathrm{~h}$ invasiv beatmet (- Tab. 1 und 2). Er zeigte ein prolongiertes Weaning und erhielt eine plastische Tracheotomie. Während der Entwöhnung von der invasiven Beatmung war es intermittierend nach einem Protokoll möglich, ihn in die Spontanatmung zu überführen. Eine 24h-Spontanatmung ohne Unterstützung des Beatmungsgeräts konnte der $\mathrm{Pa}$ tient nicht durchführen. Er entwickelte erneut eine respiratorische Verschlechterung und verstarb 6 Tage nach Beginn des Weaning-Protokolls.

Radiologische Befunde und histopathologische Korrelation. Am 33. Behandlungstag wurde der Patient zu- brosierung des pulmonalen Interstitiums (- Abb. 2h). Abdominell imponierten radiologisch Zeichen einer mesenterialen Ischämie, wobei das vollständige Kontrastierungsdefizit der Darmwand aboral des Treitz-Bands bei erhaltener Kontrastierung der A. mesenterica superior auffiel (-Abb. 3a-d). Makropathologisch bestätigte sich die mesenteriale Ischämie, und mikroskopisch war eine intramurale Perfusionsstörung mit Gefäßdilatation und -hyperämie nachweisbar (• Abb. 3e-g).

\section{Diskussion}

\section{Patient 1}

Die beobachteten Perfusionsdefizite in Lungenarealen ohne computertomographische COVID-19-typische Veränderungen, wurden durch die jeweiligen Jodkarten detektiert. Hinweise auf eine Lungenarterienembolie, ein durch Mucus Plugging bedingtes Air Trapping mit konsekutiver hypoxischer Vasokonstriktion oder verminderte Gefäßdichte durch Emphysem zeigten sich nicht. In Zusammenschau mit den mikroradiologischen und histopathologischen Befunden wiesen die Perfusionsdefizite auf ein relatives Versagen der physiologischen Vasokonstriktion der pulmonalen Endstrombahn bei Hypoxie als Ausdruck einer Schädigung der pulmonalen Mikrogefäßstrombahn hin. Dieses als Euler-Liljestrand-Mechanismus bekannte Regulationsphänomen stellt physiologisch die Perfusion von ventiliertem Lungenparenchym durch Reduzierung der Durchblutung nichtbelüfteter Areale sicher. Die gelegentlich nachweisbaren fibrinoiden Mikrogefäßthromben scheinen das Ausmaß des Perfusionsdefizits nicht hinreichend $\mathrm{zu}$ erklären. Diese Hypothese wird gestützt durch aktuelle Studien, die auf einen direkten Mikrogefäßschaden bei COVID-19 hinweisen [4, 5]. Möglicherweise ist der Mikrogefäßschaden auf die bekanntermaßen hohe Expression der Metallopeptidase Angiotensin-Converting Enzyme 2 (ACE2) auf humanem pulmonalem Gefäßendothel zurückzuführen, was eine SARS-CoV2-Infektion von Endothelzellen ermöglichen könnte [10, 11]. Ein eindeutiger 

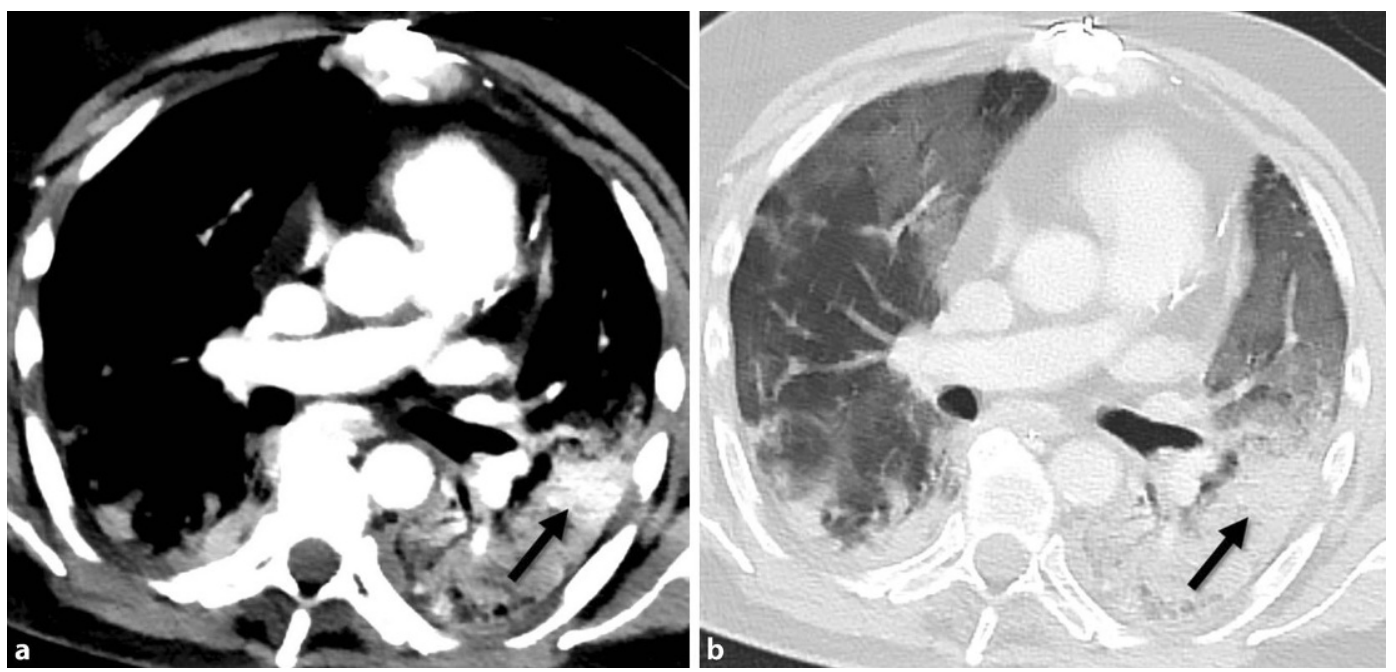

Abb. $5<$ Lokale Hyperkontrastierung einer Konsolidierung im posterobasalen linken Oberlappen in der MonoE40keV-Rekonstruktion (a) und im Lungenfenster (b) bei Patient 1, 19 Tage vor dem Versterben

Nachweis von SARS-CoV-2 in kapillaren Lungenendothelzellen und eine damit verbundene direkte Schädigung wurden bislang nicht angeführt. Kürzlich häuften sich darüber hinaus Hinweise auf eine zusätzlich hohe Expression von ACE2 auf Perizyten, eine dem Bindegewebssystem zugehörige Zellpopulation, die auf der Außenseite von Kapillargefäßen lokalisiert ist und durch ihre Kontraktibilität an der Regulierung des Gefäßlumenquerschnitts und letztlich der Perfusion beteiligt ist [12-14]. Die Zellpopulationen mit hoher ACE2-Expression sind schematisch im Kontext eines pulmonalen Alveolus dargestellt (- Abb. 4) und tabellarisch aufgeführt (- Tab. 4).

Die zum Teil kräftige Perfusion von COVID-19-typischen Lungenläsionen weist (• Abb.5) ebenfalls auf ein Versagen der hypoxischen pulmonalen Vasokonstriktion hin. Darüber hinaus könnte die Hyperperfusion von Läsionen in Zusammenhang mit der von Ackermann et al. beschriebenen aberranten Angiogenese einem lokalen Kompensationsmechanismus entsprechen, der nach Schädigung des Gefäßendothels als Antwort auf eine lokale Mikrothrombosierung sowie durch immunologische Reaktionen und Veränderungen in der mikrovaskulären Hämodynamik stimuliert werden könnte [4]. Die in der CT abgrenzbaren Perfusionsstörungen sind somit als Ausdruck der klinisch beobachteten intrapulmonalen Rechts-Links-Shunts und der Verschlechterung des Ventilations-
Perfusions-Verhältnisses anzusehen, die letztlich zur Hypoxie beitragen.

Eine Hypoperfusion in Lungenabschnitten, die CT-morphologisch COVID-19-typische Veränderungen aufweisen, wie von Lang et al. beschrieben [7], könnten entweder Ausdruck einer noch physiologischen hypoxischen pulmonalen Vasokonstriktion bei relativer Reduktion alveolarer Ventilation sein oder bereits auf eine kapillare Mikrothrombosierung hinweisen. Eine relative Hypoperfusion von Lungenabschnitten, die keine COVID-19typischen Läsionen tragen, wie im hier präsentierten Fall, stellt klinisch ebenfalls eine relevante Situation dar, da in diesem Fall der Gasaustausch gerade in jenem Lungengewebe kompromittiert ist, das von Seiten der Ventilation noch zu einer Kompensation der Hypoxie fähig wäre. Das Nebeneinander von hyperperfundierten Läsionen und minderperfundierten belüfteten Lungenarealen kann hämodynamisch auch im Sinne eines lokalen intrapulmonalen Steal-Phänomens betrachtet werden. Das Auftreten des relativ hypoperfundierten Areals ohne Milchglastrübungen oder Konsolidierungen scheint nicht lediglich Ausdruck einer normalen Perfusion zu sein, da sich in den apikalen Abschnitten des rechten Oberlappens auch Bereiche finden, die ohne Nachweis von Milchglastrübungen eine relativ höhere Perfusion zeigen (• Abb. 6). Zudem zeigte die korrelierende histopathologische Aufarbeitung, dass relativ perfusi- onsgeminderte Abschnitte strukturelle Zeichen einer Mikrogefäßschädigung aufweisen können (• Abb. 1i, j).

\section{Patient 2}

Pulmonal war eine fortgeschrittene COVID-19-Lungenerkrankung mit einem Nebeneinander von akutem Alveolarschaden, frustraner Reparation und Fibrose mit entsprechenden funktionellen Implikationen für die Blut-LuftSchranke und dem alveolären Gasaustausch zu verzeichnen. Das zudem bestehende, ausgeprägte intestinale Kontrastierungsdefizit ohne Nachweis eines Gefäßverschlusses größerer Mesenterialgefäßäste bewegte uns zur gezielten pathologischen Korrelation und Analyse der mesenterialen kapillären Endstrombahn, in der sich histologisch eine intramurale Perfusionsstörung nachweisen ließ. Wenngleich histologisch keine Mikrogefäßthrombosen nachgewiesen wurden, scheint es naheliegend, dass diese aufgrund einer möglichen umschriebenen, segmentalen Ausbildung in den angefertigten Schnittstufen nicht erfasst wurden. Diese Annahme wäre im Einklang mit der Lungenpathologie, bei der umschriebene Mikrothrombosierungen auch einen erheblichen Einfluss auf die Perfusion benachbarter Lungenabschnitte haben. Differenzialdiagnostisch könnte die Darmischämie auch als Folge einer Low-output-Symptomatik bei COVID-19-bedingtem kardiopulmonalem Versagen zurückgeführt werden. Inwie- 

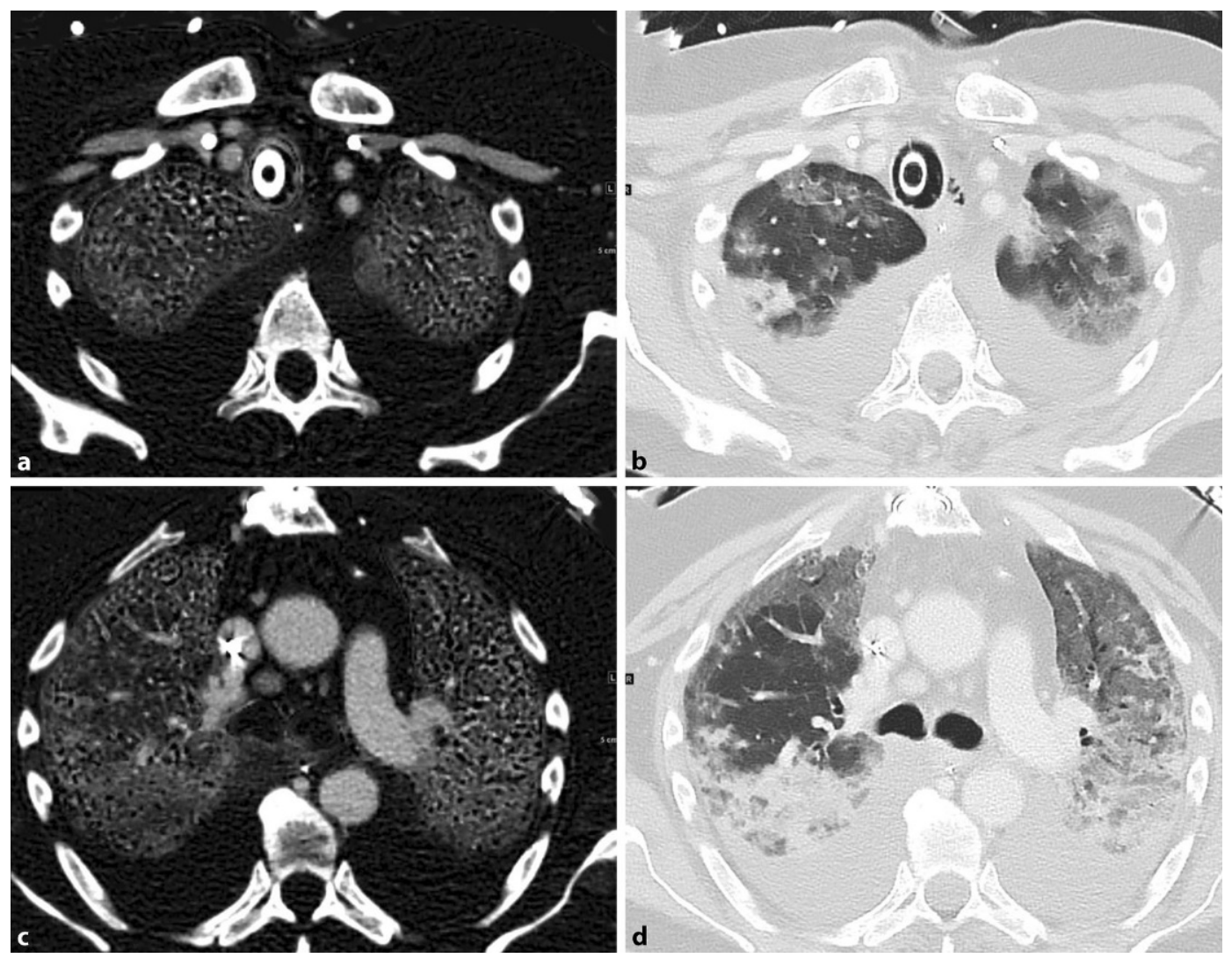

Abb. $6<$ Vergrößerte Ansicht der korrespondierenden Schichten 2 und 6 in der Jodkarte und dem Lungenfenster (Vergrößerungen der $\bullet$ Abb. 1a, b). Schicht 2 zeigt Areale ohne Milchglastrübungen/ Konsolidierungen mit erhaltener Perfusion $(\mathbf{a}, \mathbf{b})$. Schicht 6 zeigt Areale ohne Milchglastrübungen/ Konsolidierungen mit relativ reduzierter Perfusion $(c, d)$

weit darüber hinaus lokale COVID19-bedingte Mechanismen einer intestinalen mikrovaskulären Dysregulation eine Rolle gespielt haben könnten, ist Gegenstand weiterer Untersuchungen.

\section{Fazit für die Praxis}

- Die Behandlung des akuten Atemversagens bei Patienten mit COVID19 bleibt aufgrund des noch geringen Verständnisses der zugrunde liegenden Pathophysiologie eine Herausforderung.

- Unsere Ergebnisse weisen darauf hin, dass neben der pulmonalen Mikrogefäßthrombosierung eine funktionelle Vasodysregulation benachbarter Lungenabschnitte bei der COVID19-Pneumonie auftritt. Diese könnte durch einen kompromittierten Euler-Liljestrand-Mechanismus erklärt werden.

- Zur abschließenden Bewertung scheinen weitere skalenübergreifende Struktur- und Funktionsanalysen der Gefäß- und Perfusionsveränderungen bei Patienten mit COVID-
19 durch die bildgebenden diagnostischen Disziplinen dringend erforderlich.

\begin{tabular}{l} 
Korrespondenzadresse \\
$\begin{array}{l}\text { W. L. Wagner } \\
\text { Klinik für Diagnostische und } \\
\text { Interventionelle Radiologie, } \\
\text { Universitätsklinikum } \\
\text { Heidelberg } \\
\text { Im Neuenheimer Feld 110, } \\
\text { 69120 Heidelberg, } \\
\text { Deutschland } \\
\text { willi.wagner@uni- } \\
\text { heidelberg.de }\end{array}$ \\
\hline
\end{tabular}

\section{Einhaltung ethischer Richtlinien}

Interessenkonflikt. W.L. Wagner, K. Hellbach, M.O. Fiedler, G.A. Salg, E. Wehrse, C.H. Ziener, U. Merle, C. Eckert, T.F. Weber, W. Stiller, M.O. Wielpütz, C. Dullin, H.G. Kenngott, H.-P. Schlemmer, M.A. Weigand, P.Schirmacher, T. Longerich, H.-U. Kauczor, F. K. Kommoss und C. Schwab geben an, dass kein Interessenkonflikt besteht.

Die Studie wurde vorab von der Ethikkommission der Medizinischen Fakultät der Universität Heidelberg genehmigt (Nr. S-242/2020). Alle beschriebenen Un- tersuchungen am Menschen oder an menschlichem Gewebe wurden mit Zustimmung der zuständigen Ethikkommission, im Einklang mit nationalem Recht sowie gemäß der Deklaration von Helsinki von 1975 (in der aktuellen, überarbeiteten Fassung) durchgeführt.

\section{Literatur}

1. Gattinoni L, Coppola S, Cressoni M, Busana M, RossiS, ChiumelloD(2020)COVID-19 does notlead to a "typical" acute respiratory distress syndrome. Am J Respir Crit Care Med 201(10):1299-1300

2. Gattinoni L, Chiumello D, Caironi P, Busana M, RomittiF,BrazziLetal(2020)COVID-19pneumonia: different respiratory treatments for different phenotypes? Intensive Care Med. https://doi.org/ 10.1007/s00134-020-06033-2

3. KlokFA, KruipMJHA, van derMeerNJM, Arbous MS, Gommers DAMPJ, Kant KM et al (2020) Incidence of thrombotic complications in critically ill ICU patients with COVID-19. Thromb Res 191:145-147

4. Ackermann M, Verleden SE, Kuehnel M, Haverich A, Welte T, Laenger F et al (2020) Pulmonary vascular endothelialitis, thrombosis, and angiogenesis in covid-19. N Engl J Med. https://doi.org/10.1056/ NEJMoa2015432

5. Kommoss FKF, Schwab C, Tavernar L, Schreck J, Wagner WL, Merle U et al (2020) The pathology of severe COVID-19 related lung damage-mechanistic and therapeutic implications. Dtsch Arztebl. https://doi.org/10.3238/arztebl. 2020.0500

6. Rubin GD, Ryerson CJ, Haramati LB, Sverzellati N, Kanne JP, Raoof S et al (2020) The role of chest imaging in patient management during the COVID-19 pandemic: a multinational consensus 
statement from the Fleischner Society. Radiology 296:172-180

7. Lang M, Som A, Mendoza DP, Flores EJ, Reid N, Carey D et al (2020) Hypoxaemia related to COVID19: vascular and perfusion abnormalities on dualenergy CT. Lancet Infect Dis. https://doi.org/10. 1016/S1473-3099(20)30367-4

8. Mai C, Verleden SE, McDonough JE, Willems S, De Wever W, Coolen J et al (2017) Thin-section CT features of idiopathic pulmonary fibrosis correlated with micro-CT and histologic analysis. Radiology 283(1):252-263

9. Neubert L, Borchert P, Shin HO, Linz F, Wagner WL, Warnecke $G$ et al (2019) Comprehensive threedimensional morphology of neoangiogenesis in pulmonary veno-occlusive disease and pulmonary capillary hemangiomatosis. J Pathol Clin Res 5(2):108-114

10. Hamming I, Timens W, Bulthuis MLC, Lely AT, Navis GJ, van Goor H (2004) Tissue distribution of ACE2 protein, the functional receptor for SARS coronavirus. A first step in understanding SARS pathogenesis. JPathol 203(2):631-637

11. Wiersinga WJ, Rhodes A, Cheng AC, Peacock SJ, Prescott HC (2020) Pathophysiology, transmission, diagnosis, and treatment of coronavirus disease 2019 (COVID-19): a review. JAMA. https://doi.org/ 10.1001/jama.2020.12839

12. Cardot-Leccia N, Hubiche T, Dellamonica J, BurelVandenbos F, Passeron T (2020) Pericyte alteration sheds light on micro-vasculopathy in COVID-19 infection. Intensive Care Med. https://doi.org/10. 1007/s00134-020-06147-7

13. Chen L, Li X, Chen M, Feng Y, Xiong C (2020) The ACE2 expression in human heart indicates new potential mechanism of heart injury among patients infected with SARS-CoV-2. Cardiovasc Res 116(6):1097-1100

14. Ziegler CGK, Allon SJ, Nyquist SK, Mbano IM, Miao VN, Tzouanas CN et al (2020) SARSCoV-2 receptor ACE2 is an interferon-stimulated gene in human airway epithelial cells and is detected in specific cell subsets across tissues. Cell 181(5):1016-1019

\section{Künstliche Intelligenz klassifiziert Darmkrebs anhand von IR- Imaging}

Mithilfe eines auf Quanten-Kaskaden-Lasern basiertem Infrarot (IR)-Mikroskopes gelang es in binnen ca. 30 Minuten, mit großer Treffsicherheit verschiedene Tumortypen zu unterscheiden. Die Klassifizierung ist die Grundlage einer Prognose des Krankheitsverlaufs und der Wahl einer Therapie.

Bei Dickdarm- und anderen Krebserkrankungen unterscheidet man zwischen mikrosatellitenstabilen (MSS) und mikrosatelliteninstabilen (MSI) Tumoren. Mikrosatelliten sind meist funktionslose, kurze DNA-Sequenzen, die sich häufig wiederholen. Patientinnen und Patienten mit MSI-Tumoren haben eine deutlich höhere Überlebensrate. Der Grund liegt in einer rund 1.000-fach erhöhten Mutationsrate der Krebszellen, die ihr Wachstum weniger erfolgreich macht. Weiterhin ist bei Patienten mit MSI-Tumoren die innovative Immuntherapie erfolgreicher. „Es ist also für die Prognose und die Entscheidung für eine Therapie wichtig zu wissen, um welche Art des Tumors es sich handelt", so Prof. Dr. Anke Reinacher-Schick, Leiterin der Abteilung für Hämatologie und Onkologie des RUB-Klinikums St. Josef-Hospital. Bisher erfolgt die differentielle Diagnose durch immunhistochemische Färbungen von Gewebeproben mit anschließender aufwändiger Genanalyse.

Schnelle und zuverlässige Messung

Das Potenzial des IR-Imaging als diagnostisches Werkzeug zur Klassifizierung von Gewebe, die sogenannte Label-freie digitale Pathologie, hatte die Gruppe um Prof. Dr. Klaus Gerwert vom RUB-Lehrstuhl für Biophysik schon in früheren Studien gezeigt. Das Verfahren erkennt Krebsgewebe ohne vorherige Färbung oder andere Markierung und funktioniert daher auch automatisiert mithilfe künstlicher Intelligenz. Im Gegensatz zur herkömmlichen differentiellen Diagnose des Mikrosatellitenstatus, der etwa einen Tag dauert, benötigt das neue Verfahren dafür nur etwa eine halbe Stunde.

Die entscheidende Verbesserung des Verfahrens liegt darin, dass das Team der Proteinforschung es auf die Erkennung einer molekularen Veränderung des Gewebes erweitert hat. Zuvor waren nur morphologische Visualisierungen des Gewebes möglich.

\section{Machbarkeitsstudie ermutigt}

In Zusammenarbeit mit dem Institut für Pathologie der RUB unter Leitung von Prof. Dr. Andrea Tannapfel und der Abteilung für Hämatologie und Onkologie des RUB-Klinikums St. Josef-Hospital führte das Forschungsteam eine Machbarkeitsstudie mit 100 Patientinnen und Patienten durch. Sie zeigte eine Sensitivität von 100 Prozent und eine Spezifität von 93 Prozent: Alle MSI-Tumoren wurden mit dem neuen Verfahren korrekt klassifiziert, wenige wurden fälschlich als MSI-Tumoren erkannt. Nun startet eine erweiterte klinische Studie, die an Proben aus der ColopredictPlus-2.0-Registerstudie durchgeführt wird. Die Registerstudie wurde von Andrea Tannapfel und Anke Reinacher-Schick initiiert und erlaubt die Validierung der Ergebnisse der veröffentlichten Arbeit. „Interessant ist die Methodik auch, weil sehr wenig Probenmaterial verbraucht wird, was in der heutigen Diagnostik mit immer mehr anwendbaren Techniken ein entscheidender Vorteil sein kann", so Andrea Tannapfel.

Ein weiterer Schritt in Richtung personalisierte Medizin

Künftig soll das Verfahren in den klinischen Workflow eingebracht werden, um herauszufinden, wie groß sein Potenzial für die Präzisionsonkologie ist. „Eine schnelle und präzise Diagnostik ist aufgrund der immer gezielteren Therapie bei onkologischen Erkrankungen von großer Bedeutung", erklärt Anke Reinacher-Schick.

Originalpublikation: Angela KallenbachThieltges, Frederik Großerueschkamp, et al: Label-free, automated classification of microsatellite status in colorectal cancer by infrared imaging, in: Scientific Reports, 2020, DOI: 10.1038/s41598-020-67052-z

Quelle: PM 23.06.2020, RuhrUniversität Bochum 